\title{
Robosonic: Randomness-Based Manipulation of Sounds Assisted by Robots
}

\author{
Filipe Costa Luz, Rui Pereira Jorge, and Vasco Bila \\ movlab - University Lusófona \\ Av. do Campo Grande, 376 \\ 1749-024 Lisbon \\ +351 217515500 ext: 2389 \\ \{filipe.luz, ruip.jorge, vasco.bila\} @ulusofona.pt
}

\begin{abstract}
In this text, we intend to explore the possibilities of sound manipulation in a context of augmented reality (AR) through the use of robots. We use the random behaviour of robots in a limited space for the real-time modulation of two sound characteristics: amplitude and frequency. We add the possibility of interaction with these robots, providing the user the opportunity to manipulate the physical interface by placing markers in the action space, which alter the behaviour of the robots and, consequently, the audible result produced. We intend to demonstrate through the agents, programming of random processes and direct manipulation of this application, that it is possible to generate empathy in interaction and obtain specific audible results, which would be difficult to otherwise reproduce due to the infinite loops that the interaction promotes.
\end{abstract}

Keywords: Augmented Reality, Robots, Sound, Agents, Randomness, Communication, Collaborative Composing

\section{Introduction}

Robosonic is an interactive application that seeks to manipulate sounds through interaction between people and robots. In the action space, a limited area of 80 x 60 $\mathrm{cm}$, one or two Khepera robots move according to random programming. In the action space, there are one or two markers, objects with which the robot may collide and which may be moved from a given position by the user. Two axes ( $\mathrm{x}$ and $\mathrm{y})$ are considered in the robot's movement. The horizontal $\mathrm{x}$-axis corresponds to frequency. If the robot is directed to the left (negative values on the $\mathrm{x}$-axis), this value decreases; if the robot is directed to the right, the value increases. By prior determination, this increase and reduction does not exceed audible limits (20 to $20,000 \mathrm{~Hz}$ ). Amplitude is considered on the vertical y-axis. Provided that the robot moves positively along the y-axis, the amplitude is increased; otherwise, it decreases. It was determined that if the robot approaches zero on the y-axis, sound will cease completely. The markers, which continue to be deployed by the user, fulfil two functions: they change the course of the robots provided that they detect the 
markers and change the tones that are to be reproduced. Every time that a tone is heard - provided that the robot finds a marker - this tone changes in a random fashion, based upon the library of effects previously loaded onto the application. Accordingly, we see the possibility of a simultaneous manipulation of three sound characteristics: the frequency and amplitude according to the robot's random behaviour in the action space and the tone according to the robot's collisions with the markers.

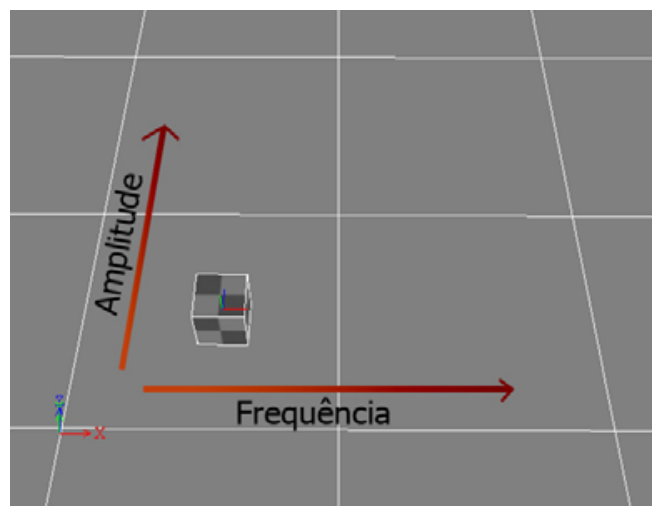

Fig. 1. Transformations in $\mathrm{X}$ and $\mathrm{Y}$

The intended result is the modulation of sounds through the robot-designed course, which will depend upon human intervention (position of markers A and B). The user provides the markers with which the robots interact in the action space, thereby altering the existing sounds in a database loaded with passive sounds to be continually executed in audible loops. The objective is the creation of a space with multiple possible audible transformations that may generate unusual results.

This does not pertain to using the robot as a generator of sounds, or rather, as a synthesizer. The main aspect in this application's development is the use of robot behaviour as a form of sound manipulation. In this project, the sounds precede the application as they were synthesized on other platforms. What this application develops is the form of a robot, its random behaviour and the user's intervention in the possibilities of altering such random behaviour, producing manipulations of specific sound.

This application anticipates the possibility of an audio recording of the entire process. This means that the results obtained may be used subsequently in other contexts. In this document, we will refer to the possibility of using these modulations created during the process in later sampling processes, to then be included in other compositions.

Since 1863, when Henri Fourneaux invented a way to programme musical execution on a piano, which he called Pianista and that would later become Pianola, 
invented in 1876 by Edwin Votey, and which includes the possibility of a piano being able to execute sequences of previously determined notes on perforated tape, numerous robotic attempts at musical execution have been witnessed. An interesting development has recently been verified, which spans diverse areas such as academics, the arts, business and teaching. The level of complexity and development is great, allowing the robots to analyze, play and even compose music. However, we take the application presented in this document as a point of departure, especially the robot's role as interface and controller in which the user will elicit a specific type of behaviour in the robot with the deployment of markers. Since the control that the user exercises over the robot is far from complete - and even minimal - it is nonetheless an aspect that especially allows this application to explore the random possibilities of manipulation. We also discuss the controller so that we can compare the situation to that of a MIDI controller, such as a keyboard, for example, which serves to transmit information pertaining to frequency, amplitude, tone and duration. The robot will transmit information regarding frequency and amplitude through its course and tone through its collision with the markers. Accordingly, we underscore the idea that this phase does not pertain to generating sounds, but specifically to establishing a synthesis process. It is applied before manipulation, through the alteration of previously existing material, namely the looped tones previously loaded on a database.

An important aspect to consider is the fact that the results obtained with this application lead us back to hearing sound specificity, given that what is heard is always unexpected in the sense that the robot produces it. John Cage, a $20^{\text {th }}$ century American composer and pioneer in the use of random processes in musical composition, paid particular attention to this possibility of us hearing basic sounds, avoiding any type of previous qualification. "The sounds enter the time-space centred within themselves, unimpeded by service to any abstraction, their 360 degrees of circumference free for an infinite play of interpretation." [1]

What is important to consider here, regarding the process of chance, is that it is more than simply randomness. It is also interesting to examine any type of imperfection, for example, that would otherwise not be obtained. "I myself use chance operations, some derived from the I-Ching, others from the observation of imperfections in the paper upon which I happen to be writing." [2]

\section{Development of Application}

The entire application was developed over a virtools platform based upon an augmented reality (AR) VirtoolKit library, VSL programming and webbots (robots) programming. A Mini DV video camera was used for video recognition (Tracking), in addition to two Khepera (K-team) robots and an interaction area. In support of the application, there is a sound library from which the robot will search for base loops.

The application is based on an AR mechanism, thereby catching a glimpse of a new visual dimension over the application to be subsequently developed. In this first 
stage, the AR allows us to detect the position of markers (interface manipulated by the user) and the robot's movements (artificial agent), thereby functioning as a tracking system for video processing. The marker's coordinates are then obtained in real time for use as frequency and volume variables for a specific sound.

Depending on the size of the interaction area, the factor that transforms the amplitude and the frequency will need to be adjusted. In the example presented, we multiplied the values of $\mathrm{X}$ and $\mathrm{Y}$ by 0.1 , in order to better capture this application's dimensions ( $60 \times 80 \mathrm{~cm})$.

In figure 2, we can analyze the schematic programming view for the transformation of the $\mathrm{x}$-axis (frequency) developed in Virtools.

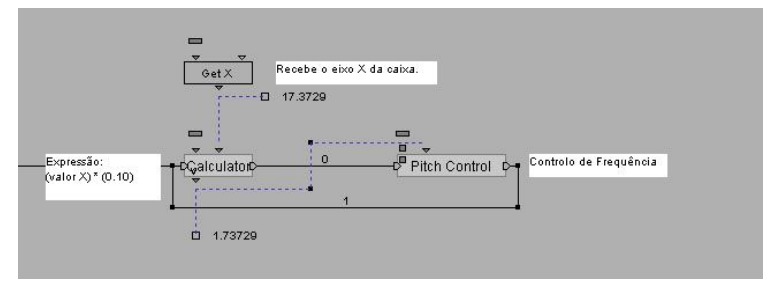

Fig. 2. Schematic view of $\mathrm{X}$ transformations

According to the structure, this code is added to the Robot, while the remaining markers are placed to determine collisions with the robot and, consequently, require it to travel new paths within the interaction space. Provided that the robot detects a marker, the sequence of sounds associated with the database is reordered, replacing the tone that the robot is about to produce with another chosen randomly in the database, composed of a library of 12 sounds essentially with the character of texture. Meanwhile in the two-robot application version, it is associated with a second library with 12 sounds of a more rhythmic nature.

\section{Robot agency}

During this interaction between the user and the robot, the intent is to blur the boundary between programming and the programmer given that in the space of this "musical game", the previous code in Virtools is merged with the robot's programming upon interaction with the user.

This does not apply specifically to studying the cyborg symbiosis between computers and humans, which occurs through the gaming experience [3], enhancing the human characteristic of attraction to the machine. At this moment, it is more important to emphasize that this type of application shows that humans "think" as agents, providing equalization between natural and artificial reasoning [4]. 
In the Robosonic application, we try to understand how the machine processes in order to seek control over its movements. It is through agency that the illusion of this human-computer communication process becomes stronger, given that the illusion that we are communicating with similar beings potentially increases the notion of "being present" in this interaction space [5].

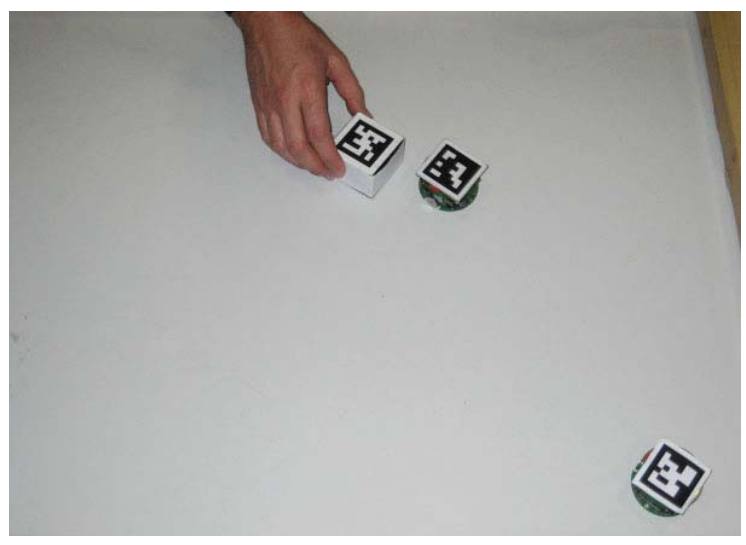

Fig. 3. User chaging fixed obstacle position to interact with two Khepera mobile robots

According to Lahti, learning programming logic to construct computer games, where we may include other types of digital spaces such as software with a graphical interface, reveals a cyborg conscience that Ted Friedman indicates as a human extension of computational processes [6]. It pertains not only to understanding the machine, but also to working in unison with it in order to create. If, on one hand, the user tries to dominate the movements and sounds produced by the robot, then on the other hand the programming that the application's creator previously developed, trying to virtualize all possible operations in the "game" space, will be updated, or rather, the programmer creatively merges with the programming and hardware to produce this interactive space.

The programming ceases to be limited to the creation of a totally defined space, where the programmer, in a strategic and virtually ludic manner, projects a space for the user to experiment and subsequently create. It does not pertain solely to "thinking like a machine" but rather to thinking with the machine. "Are we using the computers, or are the computers using us?" [7]. Considering programming loops, John Maeda questions how far the computer will go without our direct interaction. Machines that function in constant cycles and that are provided with the ability to memorize may be considered autonomous and of an artificial nature, yet still the product of human technology. 
Robosonic is therefore the materialization of an "inhabitable world" in the sense that it will be occupied by robots, bots, users and spectators. As a usual task, the intent to control this small world and, likewise, all of the restlessness that the lack of control may generate, is notable. Such a phenomenon occurs because the game space is inhabited by other agents who adapt to our form of interaction [8], trying to avoid control because of previous programming, or because they react in an apparently unpredictable manner due to "errors" in code.

The robot functions as an agent that seeks to move freely in the action space, interacting with the database that is reordered through a random programming factor and from the contact between the markers and the robot, or due to the detection of another robot. In this first phase, these agents present artificial intelligence at an elementary level, since they are limited to repositioning themselves in space (path finding) provided that their paths are marked by obstacles (markers). However, upon initial testing, it was immediately deduced that the agents completely revolutionized the application interface, creating a new paradigm for human-machine communications. A space inhabited by artificial beings that reacts with human interaction seems to provide personality to software [9] and generate special attention. When simulations of movement are shown to be synchronized with image and sound, they generate a type of "special attraction" over the audiences [10].

As an accepted definition, agents present their own properties [11] identified as: autonomy, by performing functions in a controlled manner without direct intervention by humans or other agents. They must respond to third-party elements whenever these contact them, while maintaining their individual objectives as a rule, conferring upon them autonomy, social aptitude and the ability to adapt to the surrounding environment, responding to transformations that may occur. Therefore, the agents must not be predictable in the performance of their tasks, demonstrating the sense of opportunity to surprise the user. To us, this concept seems to be an excellent reason to work towards the creation of a communications interface hypermediated by software, hardware and human agents, for the construction of sounds that cannot in any way be controlled exclusively by the user. Therefore, the agents have a fundamental role in the lack of control over manipulation, and, accordingly, in seeking unexpected paths that would carry us to unique musical results, abstracting the user through the transparency of connection that occurs in this interface of human-machine interaction.

\section{Sound Manipulation}

Above all, this application must be seen according to two different perspectives: sound manipulation and collaborative composing.

What should be observed in manipulation is the manner in which the course which the robot takes in a specific space provides modulation of the two sound characteristics, associated with the two axes of movement: on the horizontal x-axis, frequency, and on the vertical y-axis, amplitude. In the case of frequency, the 
application provides a central area in which the sound is reproduced with the base frequency and so forth. Whenever there is movement to the left, the sound becomes deeper, while in moving to the right, the sound becomes sharper. The programming initially defined that both the deep and sharp extremes will always be audible sounds, i.e., sounds that fall within the audible human range (between 20 and 20,000 $\mathrm{Hz}$ ). In the case of the vertical axis, the possibility is assumed of sound going from zero - absence of sound - up to the application's maximum permitted volume. Given the application's functional type, fine tuning was intentionally set aside. What matters most is what is heard without concern for integration of that sound in the context of a scale according to musical notes.

The audible material is what matters.

"Ultimately, however, musical instruments, scales, and tuning systems are only the material and conceptual infrastructure onto which musical style is built. They may, in part, determine what sounds are played, but they have much less influence on how they are played." [12]

Another type of interface, such as MIDI controllers, allows for the manipulation of parameters along with frequency (notes). What emerges particularly and uniquely is that the robot's random movement in space suggests results in the context of unique amplitude / frequency combinations.

And, it is in this way that the issue of interface demonstrates interest in the context of this application.

"If a composer sets up a process which allows each player to move through the material at his own speed, for example, it is impossible for him to draw things together into some kind of calculated image, a particular effect or pattern of logical connections." [13]

Experimentation in the context of sound manipulation must be taken into account as the determining factor in this process. Beyond conducting the application as a tool, for the purpose of obtaining specific results - always conditioned given the fact that we are using this tool and that by nature it leaves its record of the result obtained - it is important to verify what such application has to offer us and that it has a unpredictable character: how can it surprise us?

By experimentation in this context, we mean this tool's attempt to take us to the limit in order to provide us with unexpected results.

"Experimental composers have evolved a vast number of processes to bring about 'acts the outcome of which are unknown' (Cage). The extent to which they are unknown (and to whom) is variable and depends on the specific process in question. Process may range from a minimum of organization to a minimum of arbitrariness, proposing different relationships between chance and choice, presenting different kinds of options and obligations". [14]

In this idea of experimentation, it is important to strengthen the concept of randomness and indetermination: these are critical to bringing about innovation. This statement of Qubais Gazala is characteristic of his practices of manipulation and alteration of musical instruments and other devices so as to infringe upon, as he says accordingly, the predictability of such instruments and devices as they are made available to the public: "We have now entered a world where music no longer 
adheres to human presumption in theory, circuit design and composition. Thus, great new sounds and musical realities can occur as one sits with one's out-of-theory instrument, a truly alien instrument, and listens to its metamorphosed output." [15]

At this level, we are particularly interested in raising the issue here of randomness in musical composition. The subject is not new, as it has been addressed many times in the history of music. Meanwhile, recent technological developments have permitted a broadening of this type of practice. Various hypotheses and methodologies may be considered here. In the particular case of the application, it would be interesting for us to call upon some of the assumptions considered by John Cage. Specifically, the issue is the presence of change in the compositional process. Perhaps what interests us most here is in the form of the unexpected. Unexpected because what the robot is going to produce with previously defined sounds on database translates into hearing unexpected effects. A certain type of $\mathrm{x}$ - and $\mathrm{y}$ combinations could be attained in some other way with some degree of difficulty. If this adds up to the possibility that someone interacts with the robot by placing the markers in a specific position in the action space, within which the sounds will be altered, then variables increase and the possibility of unexpected results increases as well. "I was exploring chance electronics. While simple, the process is explosive in its startling audio output. Fantastic aleatoric music might result composed of either 'real' instruments (samples) or layers of evolving indefinable sounds" [16]

As a composer, John Cage occupied an illustrious position as regards the use of experimental musical composition processes throughout the $20^{\text {th }}$ century. The concept of chance in musical composition is particularly important in this composer's work. He asserted that chance was completely legitimate as a compositional process. To him, in order for indetermination to be obtained, whether with traditional musical instruments, or through devices created for the purpose of proceeding with that type of manipulation, rules for indetermination in the context of contemporary composition need to be recognized. Cage clearly rejected a certain stubbornness on the part of many composers who did not consider that chance was part of the glossary and praxis of composition. To Cage, such rigidity of processes in seeking to group, include and catalogue everything is tragic and creatively inhibiting. This is especially the case because it rules out obtaining innovative results, which, de rigeur, should be part of any composer's guidelines.

The concept of collaborative composing plays an important role here. Above all, it pertains to verifying how the robot, as a technological device, facilitates this collaborative composing. At an elementary level, when two people interact with their instruments, they are already in a situation of collaborative composing. But the central point here is the collaboration between the behaviour of robots and whoever interacts with them. It pertains to collaborative composing to the extent that the robots offer a part of their material: their specific behaviour in the space that allows for a specific modulation of sounds. In turn, the users who interact with the robot, changing the position of the markers with which the robot collides, so prompting it to activate other sounds, represent the other part of material that is brought together in this collaboration. This allows the user to always have a margin for error - he knows, for example, that if he places the marker in front of the robot, 
the robot will run into it and change the sound. Likewise, he knows that if he moves the marker away from the robot, the robot will not collide with it and the same sound will persist. Nonetheless, the possibility of the user's control is limited by the unpredictability of the robot's random behaviour. The article of Kapur, A.; Eigenfeldt, A.; Bahn, C. and Schloss, A. should be consulted in this regard. Regarding collaborative musical composing using robots, it states: "The goal of many of the predecessors in the academic and artistic circles who build musical robotic systems has been to design new instruments to Express new musical ideas, not attainable by audio speakers, or human performers." [17]

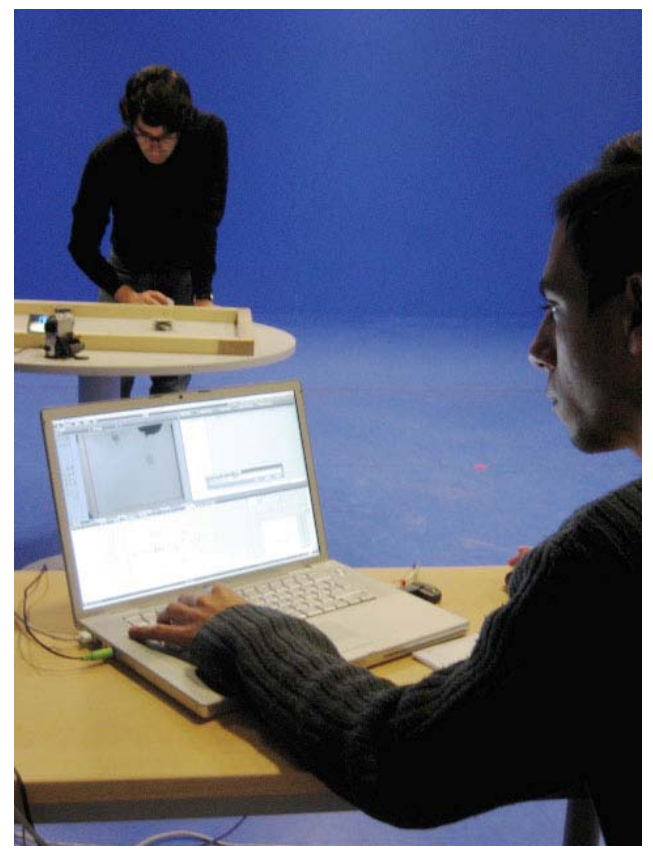

Fig. 4. Augmented Reality control PC providing randomess sound libraries for musician interact.

With the first impulse in the sense of industrial production, the domain of robotics has expanded to other areas such as entertainment, for example. The use of robots in contexts of musical execution and creation are undergoing further development. We may even confirm that, within the arts world, music has exhibited the broadest use of robots. Certain characteristics contribute to this impact, namely the fact that the robot provides an interesting role as an interface for musical applications, as well as the fact that music is an art produced intrinsically by creation and manipulation - i.e. sounds - an activity in which robots, given their specific characteristics, may provide a valuable contribution. 


\section{Conclusion}

The development of this application allows knowledge to be consolidated and information from different areas, such as robotics, augmented reality and musical composition, to be brought together.

Such development enables the establishment of a knowledge base that can be subsequently developed, namely through the integration of more robots, the integration of the video component related to sound manipulation developed by the application, and the interaction of other musicians and respective instruments with this application.

The possibility that the sounds manipulated by this application could be recorded for later use in other contexts should also be considered, strengthening the idea of using this application as a means rather than an end.

The performative aspect and the issue of interface are also clearly important in this study. The experience is performative, in the sense that the user, or users, are projected into a space for creation, simulation and visualization. They act at the moment of interaction and help to set into motion the action caused by the robots' movements.

Of future interest is the application's development in the context of its ability to integrate with other platforms in the creation and manipulation of sound and image.

\section{Acknowledgments}

We would like to thank Professor João MCS Abrantes and Professor Manuel Costa Leite for their support in terms of time, assistance and knowledge. We are also grateful to José Dinis and Fernando Marques for their unconditional help and to the Fundação para a Ciência e Tecnologia (Science and Technology Foundation) for supporting Project Evolutio: from Evolutionary Robotics to the Philosophy of Natural Cognition and Communication (PTDC/FIL/75060/2006).

\section{References}

1. CAGE, J. (1973), Silence: Lectures and Writings, Wesleyan University Press, p.59

2. CAGE, J. (1973), Silence: Lectures and Writings, Wesleyan University Press, p.17

3. LAHTI, M. (2003), “As We Became Machines" in The Video Game Theory Reader, pp. 157-170.

4. FRIEDMAN, T. (1999) Civilization and Its Discontents: Simulation, Subjectivity, and Space, in Discovering Discs: Transforming Space and Genre, $C D-R O M$, ed. Greg Smith, New York University Press, http://www.duke.edu/ tlove/civ.htm 
5. MURRAY, J. (1997), Hamlet on the Holodeck - The Future of narrative Cyberspace, New York, The Free Press, pp.154-182

6. LAHTI, M. (2003), "As We Became Machines” in The Video Game Theory Reader, pp. 157-170.

7. MAEDA, J. (2003), “The Infinite Loop”, in Code - The Language of Our Time, Ars Electrónica 2003, Germany, Hatje Cantz, pp.168-171

8. TVEIT, A. (2001) A survey of Agent-Oriented Software Engineering, Norwegian University of Science and Technology, http://csgsc.idi.ntnu.no/2001/pages/papers/atveit.pdf

9. JOHNSON, S. (1997), Interface Culture: How new technology transforms the way we create and communicate. 2ed. New York. Basic Books.

10. DARLEY, A. (2000), Visual Digital Culture, surface play and spectacle in new media games, London, Routledge

11. JENNINGS, J. \& WOOLDBRIDGE, M. (1996), Software Agents, IEE Review, January, pp.17-20, http://www.ecs.soton.ac.uk/ nrj/pubs.html\#1996

12. THÉBERGE, P. (2006)“Music / Technology / Practice; Music Knowlwedge in Action, p. 285 in Bennett, A.; ShanK, B.; Toynbee, J - The Popular Music studies reader, london, routledge

13. NYMAN, M. (1999), Experimental Music, Cage and Beyond, Cambridge, Cambridge University Press, p. 29

14. NYMAN, M. (1999), Experimental Music, Cage and Beyond, Cambridge, Cambridge University Press, p. 4.

15. GHAZALA, Q. (2004), "The Folk Music of Chance Electronics: CircuitBending the Modern Coconut” in Leonardo Music Journal, vol. 14, p.98

16. GHAZALA, Q. (2004) "The Folk Music of Chance Electronics: CircuitBending the Modern Coconut” in Leonardo Music Journal, vol. 14, p.98

17. KAPUR, A.; EIGENFELDT, A.; BAHN, C. e SCHLOSS, A.(2008),

“Collaborative Composition for Musical Robots in ARTECH, p.67 